\title{
'||||||||||||||||||||||||||||||||||||||||||||||||||||||||||||||||||.
}

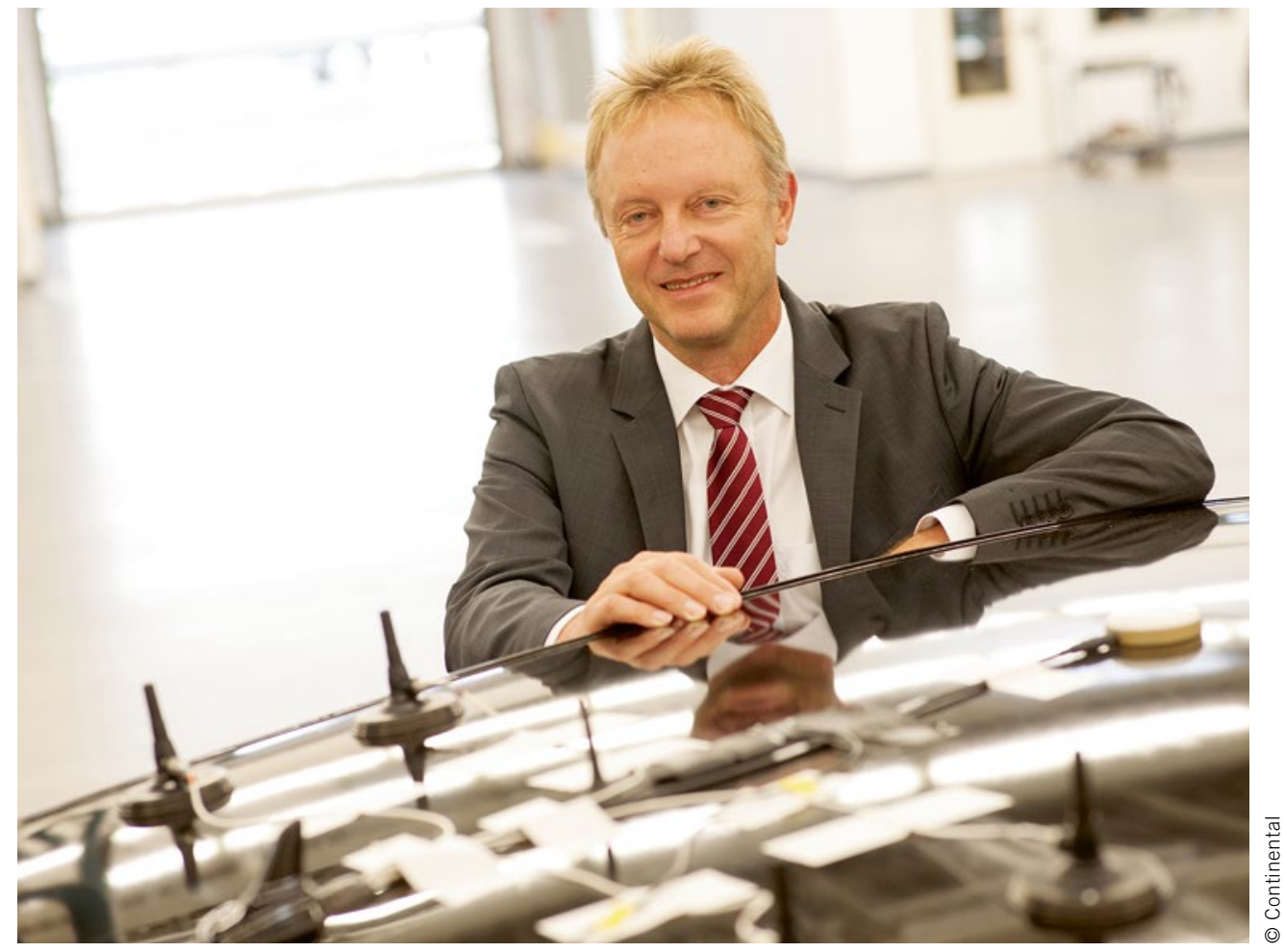

Dr.-Ing. Bernhard Klumpp

Executive Vice President, Passive Safety \& Sensorics Business Unit, Member of the Management Board, Chassis \& Safety Division,

Continental

\section{Sensors Will Protect Us in an Emergency}

The number of road deaths in Germany rose slightly in 2015, after having fallen for decades. We can help to counteract this development by interconnecting passive and active safety systems. In addition, connecting the information from different sensors to create an "intelligent" and integrated safety concept can improve vehicle and road safety even further. The resulting integral safety systems will help to provide better protection both for vehicle occupants and for more vulnerable road users. As a result, we at Continental are gradually moving closer to the vision of accident-free driving.

What does this mean in specific terms? The field of integral safety, like many other areas, is subject to constant change. Active safety sensors and systems, which help to avoid accidents, are being linked with passive safety components, which help to mitigate their consequences. The aim of this kind of networking is to obtain more information and, therefore, a more accurate picture of the vehicle's environment and the potential risks. As information suppliers, sensors form the foundation of the system, while "intelligent" control units evaluate the data and decide whether possible safety measures should be taken. This means that sensors can protect us in an emergency. Our engineers at Continental are developing increasingly intelligent systems which can be used to create an integrated safety concept.
If, despite all of this, an accident occurs, it is important to minimise its consequences, for example by means of the postcrash braking function. The systems involved ensure that after an initial impact the car is brought quickly and safely to a standstill without becoming involved in a further accident. Depending on the seriousness of the accident, the function also calls the emergency services at the same time. In the event of the car skidding, the information from the vehicle dynamics sensors is passed to the algorithm that triggers the airbags and combined with the pictures from the camera in the driver assistance system, for example. Ideally, the airbags would be inflated one by one to provide the best possible protection for the vehicle occupants.

Sensor information is not only networked inside the vehicle. Safety-related data is also exchanged between vehicles to warn following and oncoming traffic, including more vulnerable road users. Predictive systems of this kind include vehicle-to-X communication, where cars share information with one another and with the infrastructure in order to identify potentially critical traffic situations at an early stage.

Road safety can only be improved by networking systems more effectively. Exchanging information securely plays an important role in this respect. Integrated safety concepts will help to ensure that more accident victims survive. 